Société d'histoire de la révolution de 1848 et des

révolutions du XIXe siècle

$31 \mid 2005$

La "Société de 48" a cent ans

\title{
Kevin Binfield (ed.), Writings of the Luddites, Baltimore/Londres, The Johns Hopkins University \\ Press, 2004, 279 p. ISBN : 0-8018-7612-5. 49,95 dollars
}

François Jarrige

\section{(2) OpenEdition}

Journals

Édition électronique

URL : http://journals.openedition.org/rh19/957

DOI : 10.4000/rh19.957

ISSN : $1777-5329$

Éditeur

La Société de 1848

Édition imprimée

Date de publication : 1 décembre 2005

ISSN : 1265-1354

Référence électronique

François Jarrige, « Kevin Binfield (ed.), Writings of the Luddites, Baltimore/Londres, The Johns Hopkins University Press, 2004, 279 p. ISBN : 0-8018-7612-5. 49,95 dollars », Revue d'histoire du XIXe siècle [En ligne], 31 | 2005, mis en ligne le 18 février 2006, consulté le 22 septembre 2020. URL : http:// journals.openedition.org/rh19/957 ; DOI : https://doi.org/10.4000/rh19.957

Ce document a été généré automatiquement le 22 septembre 2020.

Tous droits réservés 


\title{
Kevin Binfield (ed.), Writings of the Luddites, Baltimore/Londres, The Johns Hopkins University Press, 2004, 279 p. ISBN : 0-8018-7612-5. 49,95 dollars
}

\author{
François Jarrige
}

L'histoire du luddisme, ce vaste mouvement de destructions des machines textiles qui traverse les comtés industriels de l'Angleterre entre 1811 et 1817, reste peu connue en France. Pourtant, depuis les Hammond, au début du XX'e siècle, jusqu'à Edward $\mathrm{P}$. Thompson et ses successeurs plus récents, l'histoire sociale britannique n'a cessé de questionner la signification de ces violences industrielles qui fascinèrent autant par leur ampleur que par l'originalité des modes d'action utilisés ${ }^{1}$. L'ouvrage de Kevin Binfield, professeur à l'Université de Murray (Kentucky), constitue une nouvelle étape importante dans la compréhension de ces révoltes. Avec une obstination remarquable, l'auteur a parcouru les dépôts d'archives anglais pour collecter le maximum d'écrits luddites (en tout plus de 100 textes). À partir de cet ensemble de sources unique, Kevin Binfield propose une étude originale de la dimension rhétorique et linguistique du luddisme. L'unicité de ce mouvement, par ailleurs très divers, tient d'abord à sa capacité à se nommer en utilisant la figure de Ned Ludd, un apprenti qui, le premier, aurait brisé une machine dans l'atelier de son maitre à la fin du XVIII ${ }^{e}$ siècle. C'est l'usage de ce nom propre, dans des contextes socio-économiques très différents, qui fait que l'on peut parler d'un mouvement là où, dans le reste de l'Europe, les bris de machines s'apparentèrent davantage à des soulèvements atomisés et dispersés, sans réelle cohérence entre eux. L'attention aux paroles et aux stratégies discursives utilisées par les luddites constituent, dans le contexte du linguistic turn anglais, le nouvel horizon de l'étude de ce mouvement. 
2 Dans l'introduction substantielle qui ouvre le livre, Kevin Binfield propose un remarquable tour d'horizon historiographique du luddisme et des principaux débats qu'il a suscités. Au lieu d'utiliser une documentation de seconde main basée sur la presse, les rapports des espions ou la correspondance des magistrats, il a choisi de partir d'une collection de documents émanant des ouvriers eux-mêmes et donnant à voir les mots des perdants plutôt que les jugements de ceux qui les ont vaincus. Ces écrits luddites sont extraordinairement divers; on trouve des lettres de menace adressées à des industriels, mais aussi des chants, des poèmes et des proclamations de toutes sortes. Selon Kevin Binfield, ces textes articulent trois types de discours: le pétitionnement et l'appel aux autorités en faveur d'un modèle de régulation, l'analyse économique qui promeut une véritable économie morale luddite, et une analyse politique qui s'articule autour du radicalisme. Pour autant, l'auteur ne tombe pas dans le piège d'une reconstruction factice d'un «texte» luddite qui serait déconnecté des réalités sociales et politiques locales.

3 En effet, les différents textes sont classés selon leur enracinement régional. Le livre s'organise ainsi en trois parties correspondant aux trois grandes régions affectées par les destructions ; chaque texte est précédé d'une mise en contexte rappelant les temps forts et les acteurs du mouvement. Dans la première partie, de loin la plus longue, Kevin Binfield réunit les écrits émanant des Midlands. Dans cette région dominée par l'industrie de la bonneterie, les luddites s'opposent moins à l'introduction des nouvelles machines - le métier à bras n'ayant pas connu de transformations majeures depuis le XVII ${ }^{\mathrm{e}}$ siècle - qu'à des nouvelles pratiques de travail plus intensives. Ces tricoteurs sur métier (framework-knitters) étaient organisés depuis longtemps, c'est seulement après l'échec des modes d'action constitutionnels auprès du Parlement que les travailleurs se tournent vers les destructions violentes. Les tricoteurs fondaient leurs demandes sur des chartes qui régulaient le métier et les procédés de fabrication utilisés. En utilisant le langage de la loi et de la coutume, les ouvriers des Midlands tentaient de présenter leurs revendications comme le produit légitime d'une culture de métier constituée et légale. En migrant vers les secteurs cotonniers du Nord-Ouest au cours de l'année 1812, le discours luddite ne rencontra pas un milieu capable d'intégrer dans la protestation cette rhétorique structurée par la loi et la coutume. Les tisserands à la main (handloomweavers) de Manchester et des villes cotonnières alentour constituaient en effet une population industrielle récente, sans réelle tradition corporative. Le langage du luddisme, qui se teinte ici de radicalisme et de jacobinisme, permettait aux tisserands de se constituer en corps unifié capable de se faire reconnaître par les employeurs et le gouvernement. Dans le Yorkshire enfin, ce sont les tondeurs (shearmen ou croppers), ces aristocrates de l'industrie lainière, qui brisèrent les mécaniques. Ici, le langage du luddisme émergea au terme d'une longue lutte contre les nouveaux procédés de production ${ }^{2}$. Alors que leurs statuts protecteurs avaient été abolis en 1809, les tondeurs utilisèrent le langage luddite pour redonner un peu de légitimité à leurs revendications en s'agrégeant à un ensemble plus large de protestations.

4 Comme on le voit, l'étude de Kevin Binfield ne cherche pas à retrouver une illusoire parole luddite qu'on pourrait extraire de la diversité des textes. Au contraire, il s'agit plutôt de montrer comment le luddisme s'apparente à un bricolage discursif complexe, comment les ouvriers puisent dans des répertoires, les thèmes et les outils propres à légitimer leurs revendications. 
5 Cet ouvrage se révèle en définitive d'une utilisation agréable et aisée. Muni d'un index très complet qui permet de naviguer entre les textes, agrémenté de quelques documents originaux reproduits, il intéressera à la fois l'histoire sociale, l'histoire de la littérature attentive aux écrits marginaux, mais aussi l'histoire politique soucieuse de suivre la diffusion des idéologies radicales. En partant des mots utilisés par les ouvriers eux-mêmes, Kevin Binfield parvient par ailleurs à dépasser certaines des apories dans lesquelles s'était enfermée l'historiographie du luddisme. Le silence supposé des luddites a permis les interprétations les plus contradictoires : certains, comme Edward P. Thompson, y détectant des tendances insurrectionnelles et révolutionnaires plus larges alors que d'autres, comme Malcom Thomis, insistaient sur le caractère circonscrit des revendications ouvrières ${ }^{3}$. En redonnant la parole aux acteurs euxmêmes, Kevin Binfield propose une lecture compréhensive du luddisme, seule à même de nous donner accès à l'univers intellectuel des classes populaires engagées dans les bouleversements de la révolution industrielle.

\section{NOTES}

1.. Nous nous permettons de signaler la publication en français d'une étude de synthèse sur ce mouvement de bris de machines et sur son historiographie : Vincent BOURDEAU, François JARRIGE et Julien VINCENT, Les Luddites. Bris de machines, économie politique et histoire, Paris, Éditions Ère, à paraître en 2006.

2.. Il faut citer l'étude remarquable de Adrian RANDALL, Before the Luddites. Custom, Community and Machinery in the English Woollen Industry, 1776-1809, Cambridge, Cambridge University Press, 1991, 336 p. 